1994-02-01

\title{
Electromagnetic characteristics of superquadric wire loop antennas
}

Michael A. Jensen

jensen@byu.edu

Yahya Rahmat-Samii

Follow this and additional works at: https://scholarsarchive.byu.edu/facpub

Part of the Electrical and Computer Engineering Commons

\section{Original Publication Citation}

Jensen, M. A., and Y. Rahmat-Samii. "Electromagnetic Characteristics of Superquadric Wire Loop Antennas." Antennas and Propagation, IEEE Transactions on 42.2 (1994): 264-9

\section{BYU ScholarsArchive Citation}

Jensen, Michael A. and Rahmat-Samii, Yahya, "Electromagnetic characteristics of superquadric wire loop antennas" (1994). Faculty Publications. 704.

https://scholarsarchive.byu.edu/facpub/704

This Peer-Reviewed Article is brought to you for free and open access by BYU ScholarsArchive. It has been accepted for inclusion in Faculty Publications by an authorized administrator of BYU ScholarsArchive. For more information, please contact ellen_amatangelo@byu.edu. 


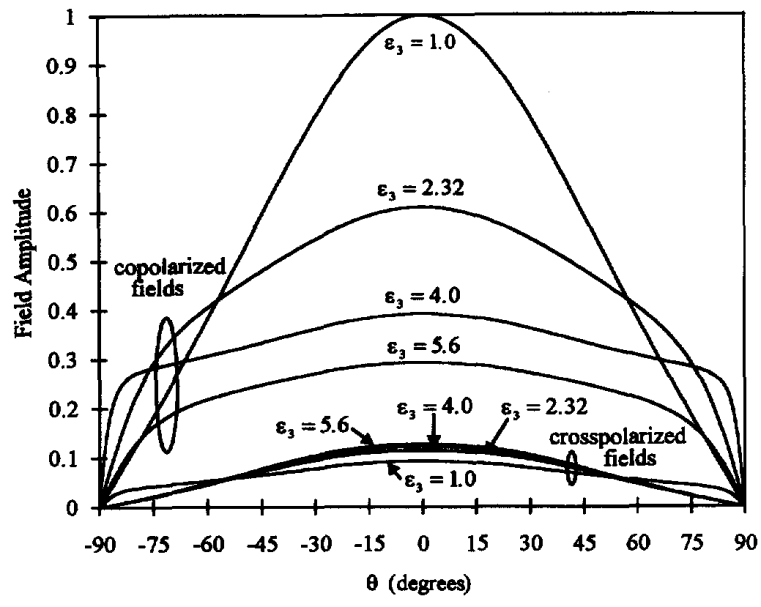

Fig. 6. The copolarized and crosspolarized patterns in $\phi=0^{\circ}$ plane for $\varepsilon_{3}=1.0,2.32,4.0$, and $5.6 ; t=0.25 \lambda_{3}, s=0$.

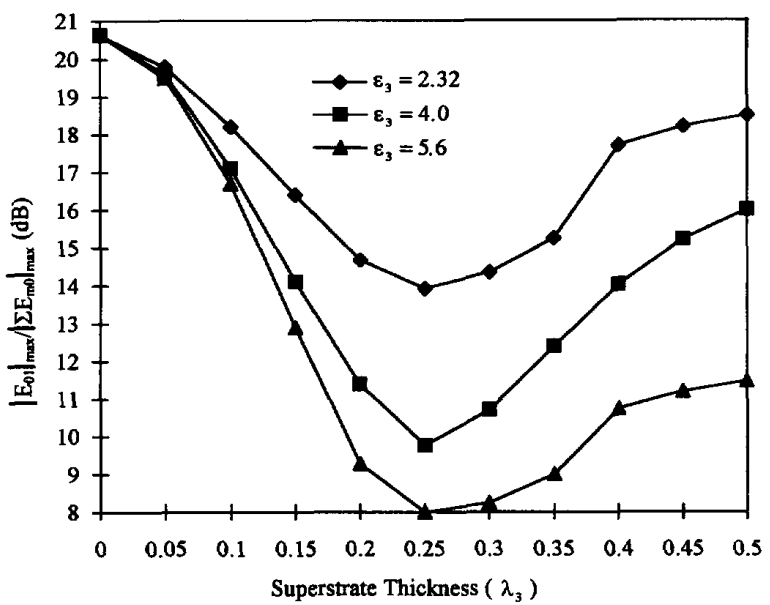

Fig. 7. Variations of the ratio of copolarized to crosspolarized fields at $\theta=0^{\circ}$ with superstrate thickness. Three cases of $\varepsilon_{3}=2.32,4.0$, and 5.6 are shown; $s=0$.

mode, the ratio of copolarized to crosspolarized fields at $\theta=0^{\circ} \mathrm{de}-$ creases with increasing superstrate permittivity and shows a minimum value when the superstrate thickness is a quarter wavelength or its odd multiples.

\section{REFERENCES}

[1] P. C. Sharma and K. C. Gupta, "Analysis and optimized design of single feed circularly polarized microstrip antenna," IEEE Trans. Antennas Propagat., vol. 31, pp. 949-955, 1983.

[2] M. D. Despande and N. K. Das, "Rectangular microstrip antenna for circular polarization," IEEE Trans. Antennas Propagat., vol. 34, pp. 744-746, 1986.

[3] K. L. Wong and S. Y. Ke, "Cylindrical-rectangular microstrip patch antenna for circular polatization," IEEE Trans. Antennas Propagat., vol. 41, pp. 246-249, 1993.
[4] R. Afzalzadeh and R. N. Karekar, " $X$-band directive single microstrip patch antenna using dielectric parasite," Electron. Lett., vol. 28, pp. $17-19,1992$.

[5] E. E. Reinhart, "Mobile communications," IEEE Spectrum, pp. 27-29, Feb. 1992.

[6] K. L. Wong and W. S. Chen, "Main-beam polarization property of a circularly polarized rectangular microstrip patch antenna," Proc. Natl. Sci. Counc. (ROC), pt. A, vol. 17, pp. 314-318, July 1993.

[7] M. L. Oberhart, Y. T. Lo and R. Q. Lee, "New simple feed network for an array module of four microstrip elements," Electron. Lett., vol. 23, pp. 436-437, 1987.

[8] T. Huynh, K. F. Lee and R. Q. Lee, "Crosspolarization characteristics of rectangular patch antennas," Electron. Lett., vol. 24, pp. 463-464, 1988.

[9] R. F. Harrington, Field Computation by Moment Method. New York: Macmillan, 1968.

[10] K. L. Wong, J. S. Row, C. W. Kuo and K. C. Huang, "Resonance of a rectangular microstrip patch on a uniaxial substrate," IEEE Trans. Microwave Theory Tech., vol. 41, pp. 698-701, 1993.

[11] J. S. Row, "Analysis of a rectangular microstrip patch in a uniaxially anisotropic multilayer medium," Ph.D. Dissertation, Dept. of Electrical Eng., National Sun Yat-Sen University, June 1993.

\section{Electromagnetic Characteristics of Superquadric Wire Loop Antennas}

Michael A. Jensen and Yahya Rahmat-Samii

Abstract-The analysis of antenna configurations in the form of a generalized superquadric loop, which includes circular, elliptical and rectangular loop geometries, is presented in this paper. Use of a Galerkin form of the moment method with piecewise sinusoidal subsectional basis and testing functions provides rapid numerical convergence and accurate representation of the antenna current. A convenient parametric representation for the superquadric curve is developed to allow a subsectional formulation using curved wire segments, rather than the commonly employed piecewise linear segments, to construct the geometry. Both magnetic frill and delta gap source models are implemented to allow a detailed study of input impedance, directivity, radiation pattern and current distribution as a function of various geometrical parameters. The results are shown to compare well with previous results for the special case of a circular loop antenna. Some useful curves are presented to aid in the design of practical superquadric loop antennas.

\section{INTRODUCTION}

With the rapid expansion in the area of mobile communications, new technology must constantly be produced to satisfy the increasing market demands. More and more these communication systems require electrically small, efficient antennas which perform well in a variety of environments. In many devices such as pagers or hand-held transceiver units, various configurations of circular or non-circular loop antennas are a suitable choice. The need to accurately understand the effects of the geometrical loop parameters on the behavior of the antenna has led to the analysis presented in this paper.

Thin wire circular loop antennas have been the focus of numerous theoretical studies over the past several decades. Most of these investigations examine the behavior of circular loop antennas by

Manuscript received September 3, 1992; revised August 30, 1993. This work was funded by ARPA Contract DAAB07-93-C-C501.

The authors are with the Electrical Engineering Department, University of California, Los Angeles, 405 Hilgard Ave., Los Angeles, CA, 90024-1594.

IEEE Log Number: 9215652. 
solving the electromagnetic boundary value problem to formulate an integral equation for the current distribution in the wire. A Fourier series expansion of the current is then used in the integral tequation and, if proper assumptions concerning the antenna excitation are made, the Fourier coefficients may be determined in closed form [1]-[9]. Although a few studies have produced reasonable results using other possible solution techniques [10]-[12], the convenience of the Fourier series current expansion appears to make it the most common choice.

Although the past work on circular loop antennas provides invaluable insight and design data for the implementation of such radiators, the recent demand for improved communications systems has led to the investigation of other possible non-circular loop antenna geometries. When attempting to extend the analysis of loop antennas to cover configurations other than the circular loop, the Fourier series expansion is no longer as convenient a choice and it becomes necessary to exploit more general solution schemes such as the moment method [13]. This approach allows the accuracy demanded by modern antenna applications while providing the flexibility required to analyze very general antenna configurations.

The analysis presented here uses the superquadric representation [14], [15] for the loop antenna to allow accurate modeling of a wide variety of different loop geometries-including the well-known circular, rectangular and folded dipole configurations-using one unified formulation. The solution utilizes the Electric Field Integral Equation (EFIE) from which the wire current distribution is obtained using a subsectional domain moment method. Instead of using existing general purpose thin-wire analysis codes which represent the geometry as a sequence of piecewise linear wire segments, we have chosen to exactly represent the loop configuration using a parametric description of the curve. This practice has been shown in [16] to lead to a more efficient computational tool. Both magnetic frill and delta gap source models are implemented to allow modeling of different possible feeding scenarios.

The analysis begins with a description of the superquadric curve and a discussion of the various variables involved. A parametric representation of the superquadric loop is then derived and applied to formulate a matrix equation from an EFIE for thin wires [17]. After a brief discussion of the source models used, the convergence properties of the approach are studied. The solution methodology is then applied to determine the current distribution and antenna parameters for several representative loop geometries including circular and noncircular loops and the folded dipole. The versatility of the analysis technique allows an in-depth investigation of the effects of the antenna shape and size characteristics on the input impedance which results in some useful design curves for many different applications.

\section{MATHEMATICAL DEVELOPMENTS}

\section{A. Superquadric Representation for Closed Curves}

A superquadric curve is the locus of points satisfying the equation

$$
\left|\frac{x}{a}\right|^{\nu}+\left|\frac{y}{b}\right|^{\nu}=1
$$

where $a$ and $b$ are the semi-axes in the $x$ and $y$ directions respectively and $\nu$ is a "squareness parameter" which controls the behavior of the loop radius of curvature. It should be noted that the form $\left[(x / a)^{2}\right]^{\xi}+\left[(y / b)^{2}\right]^{\xi}=1$ is also commonly used to describe the superquadric geometry, where $2 \xi=\nu$. Fig. 1 illustrates the effect of the squareness parameter by presenting the curve geometry for $\nu=2,3$ and 10 and an aspect ratio of $b / a=2$. As can be seen from this figure, a value of $\nu=2$ corresponds to an ellipse while the loop squareness increases with $\nu$. It is evident that the

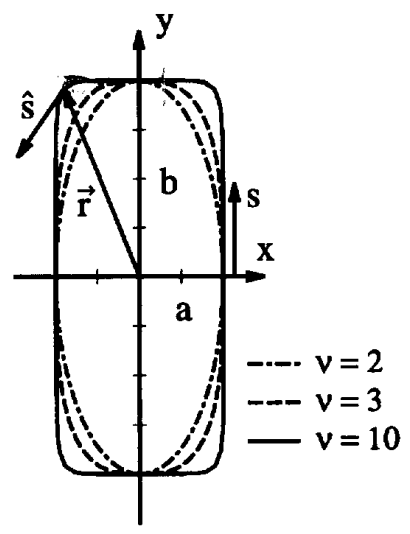

Fig. 1. Superquadric loop geometry for $\nu=2,3$ and 10 with $b / a=2$.

superquadric representation allows modeling of numerous different loop configurations through variation of the shape parameters $a, b$ and $\nu$. Also, the model's capability to provide rounded corners on otherwise rectangular loops allows accurate representation of many practical geometries. This flexibility in geometrical configuration selection can be very useful for antenna packaging considerations.

\section{B. Parametric Representation}

In order to permit the use of curved subsectional wire segments in the moment method analysis of superquadric loop antennas, it is necessary to first represent the curve in a convenient parametric form. Among the many representations possible, our investigation has shown that a form which provides mathematical simplicity and numerical stability assumes the form

$$
\begin{aligned}
& x=a \psi(\beta) \cos \beta \\
& y=b \psi(\beta) \sin \beta
\end{aligned}
$$

where

$$
\psi(\beta)=\frac{1}{\left(|\sin \beta|^{\nu}+|\cos \beta|^{\nu}\right)^{\frac{1}{\nu}}}
$$

and the parameter $\beta$ is in the range $0 \leq \beta \leq 2 \pi$. An important feature of this particular representation is the fact that equal divisions in the parameter $\beta$ result in reasonably equal values of arc length for the subsectional segments. The unit tangent vector $\hat{s}$ shown in Fig. 1 is obtained by differentiating the position vector $\vec{r}$ with respect to $\beta$ and normalizing to obtain

$$
\begin{aligned}
\hat{s}= & s_{x} \hat{x}+s_{y} \hat{y} \\
= & \frac{1}{\gamma(\beta)}\left[-a|\sin \beta|^{\nu-1} \operatorname{sgn}(\sin \beta) \hat{x}\right. \\
& \left.+b|\cos \beta|^{\nu-1} \operatorname{sgn}(\cos \beta) \hat{y}\right]
\end{aligned}
$$

where

$$
\gamma(\beta)=\sqrt{a^{2}|\sin \beta|^{2 \nu-2}+b^{2}|\cos \beta|^{2 \nu-2}}
$$

and $\hat{x}$ and $\hat{y}$ are the unit vectors in the $x$ and $y$ directions respectively. The differential arc length $d s$ may be expressed in terms of the differential $d \beta$ as in

$$
d s=\gamma(\beta) \psi^{\nu+1}(\beta) d \beta=\Delta(\beta) d \beta
$$

where $\psi(\beta)$ and $\gamma(\beta)$ are given in Eqs.(3) and (5) respectively. The loop arc length $s$ from the feed point at the positive $x$-axis to a point 
$\beta_{o}$ is computed by integrating the differential in (6) as in

$$
s=\int_{0}^{\beta_{o}} \Delta(\beta) d \beta .
$$

The total loop perimeter $P$ of course results when $\beta_{o}=2 \pi$ is used in (7). The area of the superquadric loop is given by

$$
A=a b 2^{2-2 / \nu} \sqrt{\pi} \frac{\Gamma(1+1 / \nu)}{\Gamma(1 / 2+1 / \nu)}
$$

where $\Gamma(x)$ is the gamma function.

\section{Integral Equation Formulation}

With the superquadric loop described in the parametric form of (2), we can develop the integral equations relating the currents on the wire to the excitation fields. For the loop geometry depicted in Fig. 1 we may use a form of the electric field integral equation for arbitrarily shaped thin wires [17]. The thin-wire assumption allows the approximation that the unknown current $I(s)$ is a filamentary current which flows in the axial direction only. This EFIE assumes the form

$$
\hat{s} \cdot \vec{E}^{\text {inc }}(s)=\frac{-1}{4 \pi j \omega \epsilon_{o}} \int_{0}^{P}\left[\hat{s} \cdot \hat{s}^{\prime} k^{2} I\left(s^{\prime}\right)+\frac{\partial I\left(s^{\prime}\right)}{\partial s^{\prime}} \frac{\partial}{\partial s}\right] G d s^{\prime}
$$

where

$$
G=\frac{e^{-j k R}}{R}
$$

and $k=2 \pi / \lambda$ is the wave number. $R=\left|\vec{r}-\vec{r}^{\prime}\right|$ is the distance from the observation point $\vec{r}$ to the source point $\vec{r}^{\prime}$ and $P$ is the loop perimeter. In this case, the filamentary current is placed on the wire surface at $\vec{r}^{\prime}=x^{\prime} \hat{x}+y^{\prime} \hat{y}+r_{\mathrm{o}} \hat{z}, r_{o}$ being the wire radius. The observation point coincides with the axis at $\vec{r}=x \hat{x}+y \hat{y}$ rather than the surface of the wire. This approximation provides a convenient approach for overcoming the difficulties associated with the singular integrand for coincident source and observation points.

\section{Moment Method Solution-Curved Subsections}

To apply the integral equation of (9) to the superquadric geometry, the parametric representation developed in Section II-B is used. This involves changing the functions of $s$ to functions of the parameter $\beta$ and integrating over the range $0 \leq \beta \leq 2 \pi$. An important feature of the use of this parametric construction is that curved wire segments, rather than the commonly-used piecewise-linear segments, are implemented to represent the geometry.

The moment method matrix is formed from the integral equation by dividing the curve into discrete segments at the points $\beta_{n}, n=1$ to $N$. A Galerkin moment method approach with piecewise sinusoidal subsectional basis and testing functions of the form

$$
f_{n}(\beta)=\left\{\begin{array}{cc}
\frac{\sin \left(\beta-\beta_{n-1}\right)}{\sin \left(\beta_{n}-\beta_{n-1}\right)} & \text { if } \beta_{n-1} \leq \beta \leq \beta_{n} \\
\frac{\sin \left(\beta_{n+1}-\beta\right)}{\sin \left(\beta_{n}+1-\beta_{n}\right)} & \text { if } \beta_{n} \leq \beta \leq \beta_{n+1} \\
0 & \text { otherwise }
\end{array}\right.
$$

is then implemented to create the linear system

$$
E_{m}=\sum_{n=1}^{N} K_{m n} I_{n}
$$

where

$$
\begin{aligned}
E_{m} & =\int_{\beta_{m-1}}^{\beta_{m+1}} f_{m}(\beta) \hat{s} \cdot \vec{E}^{\mathrm{inc}}(\beta) \Delta(\beta) d(\beta) \\
K_{m n} & =\frac{-1}{4 \pi j \omega \epsilon_{o}} \int_{\beta_{m-1}}^{\beta_{m+1}} d \beta f_{m}(\beta) \Delta(\beta) \int_{\beta_{n-1}}^{\beta_{n+1}} d \beta^{\prime}
\end{aligned}
$$

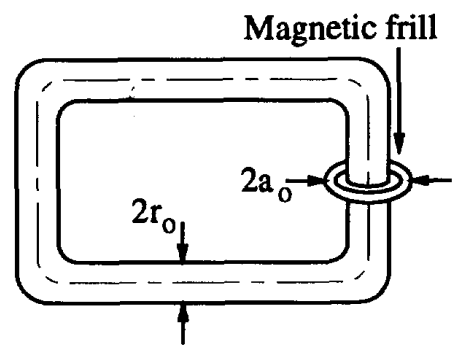

Fig. 2. Full-loop model of the superquadric loop fed by a magnetic frill source.

$$
\begin{aligned}
& \times\left\{k^{2}\left(s_{x} s_{x}^{\prime}+s_{y} s_{y}^{\prime}\right) f_{n}\left(\beta^{\prime}\right) \Delta\left(\beta^{\prime}\right)-\frac{(j k R+1)}{R^{2}}\right. \\
& \left.\left[s_{x}\left(x-x^{\prime}\right)+s_{y}\left(y-y^{\prime}\right)\right] \frac{\partial f_{n}\left(\beta^{\prime}\right)}{\partial \beta^{\prime}}\right\} \frac{e^{-j k R}}{R}
\end{aligned}
$$

for $m=1, \ldots, N$. The unknown current is then obtained from the series

$$
I(\beta)=\sum_{n=1}^{N} I_{n} f_{n}(\beta)
$$

\section{E. Source Models}

In this particular study, both delta gap and magnetic frill source models are used to allow the analysis of different feeding scenarios. In the delta gap model, the impressed field is assumed constant and to exist only in a small feeding gap in the loop conductor. For a gap of length $\Delta g$ with an impressed feed voltage of $V$, the excitation field in the gap is given by

$$
\hat{s} \cdot \vec{E}^{\text {inc }}(\beta)=\frac{V}{\Delta g}
$$

and is zero elsewhere. In general, the gap width $\Delta g$ is not constrained to equal the subsectional arc length. Although the implementation of this source model is relatively straightforward, it is generally less accurate than the frill model for input impedance computations, especially when coarse subsectioning is used or thick wires are represented.

The magnetic frill source model is designed to predict the characteristics of a half-loop over a large ground plane fed by a coaxial line through the large conducting plate. The impressed field in this case is that produced by an annular ring of magnetic current at the feed point. Using image theory, the half-loop with the ground plane can be represented as a full-loop with the magnetic frill plus its image as shown in Fig. 2. The inner radius of the annular current ring corresponds to the antenna wire radius $r_{o}$, as shown in the figure. The outer annular radius, $a_{o}$, is set to the value of the outer radius of the coaxial line feeding the antenna, which is $a_{0}=2.3 r_{o}$ for an air-filled $50 \Omega$ coaxial cable. The details of this source model are available in the literature [9], [18]. It is important to recognize that the current and input admittance of the half-loop are twice those of the full-loop antenna model of Fig. 2. In the results shown later in the paper, we present the data for the full-loop scenario.

\section{COMPUTATIONAL RESULTS}

\section{A. Convergence}

Before presenting some computational examples for specific loop geometries, the convergence properties of the numerical solution must 


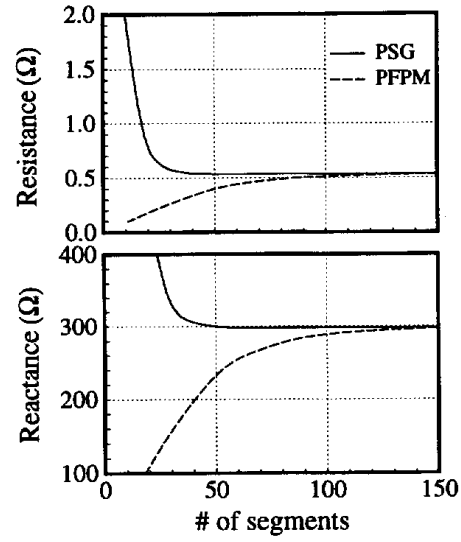

(a)

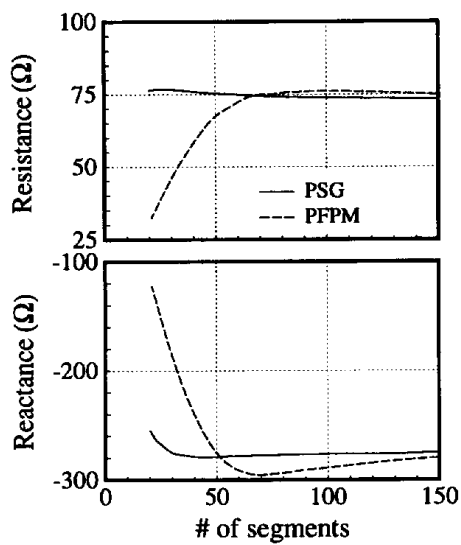

(b)

Fig. 3. Input impedance versus number of subsections for a circular loop antenna $(a=b, \nu=2, \Omega=10)$ using both piecewise sinusoidal Galerkin (PSG) and pulse-function point-matching (PFPM) approaches: (a) $0.2 \lambda$ and (b) $0.8 \lambda$ circumference.

be examined. To perform this investigation, the model was applied to a circular loop configuration ( $a=b, \nu=2$ ) with

$$
\Omega=2 \ln \frac{2 \pi a}{r_{o}}=10
$$

as a measure of the antenna wire radius. Five-point Gaussian Quadrature integration was used to evaluate the integrals in Eqs. (13) and (14) for each subsection. The magnetic frill source model was implemented for a $50 \Omega$ air-filled feeding coax. For comparison purposes, a pulse-function basis current expansion with point-matching for testing (PFPM) was implemented as well as the piecewise sinusoidal Galerkin (PSG) approach developed above. Figs. 3(a) and (b) show the convergence of the input impedance as a function of the number of subsections used for loops with circumferences $2 \pi a=0.2 \lambda$ and $2 \pi a=0.8 \lambda$ respectively for both choices of basis functions. As can be seen, both methods converge to the same stable solution, but the piecewise sinusoidal model converges significantly faster. This illustrates the tradeoff between the added programming complexity of the piecewise sinusoidal method and the increased computational costs associated with the simpler pulse-function pointmatching algorithm. The plots of Fig. 3 also serve to indicate the

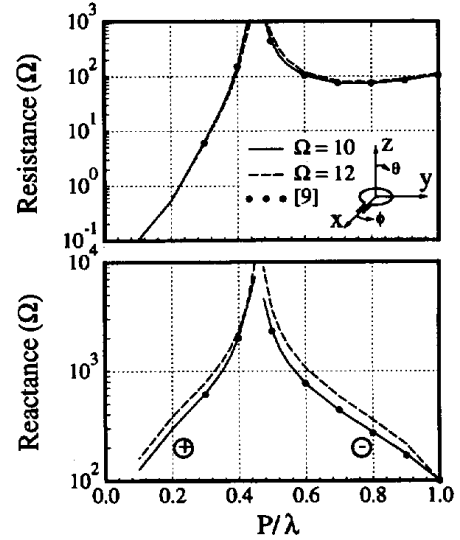

Fig. 4. Input impedance versus loop circumference $P$ for a circular loop $(a=b, \nu=2$ ) with $\Omega=10$ and 12. The dots are values from Zhou and Smith [9] provided for comparison.

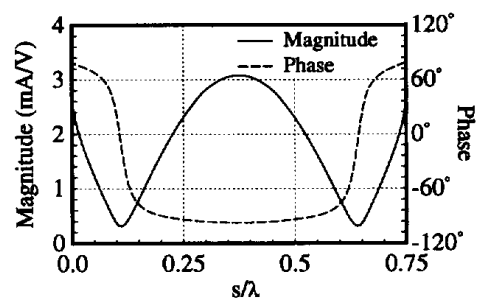

(a)
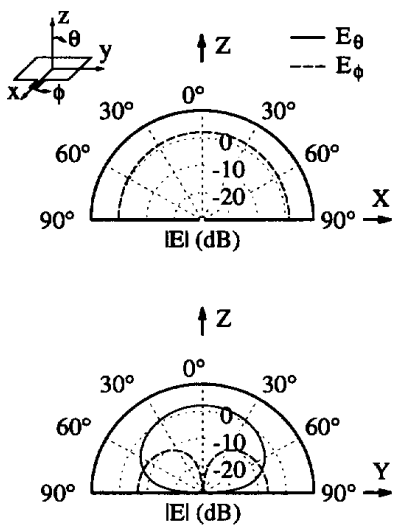

(b)

Fig. 5. (a) Current distribution (magnitude and phase) versus perimeter coordinate $s$ and (b) principal plane directivity patterns (in $\mathrm{dB}$ ) versus $\theta$ for a $0.75 \lambda$ loop with $\nu=10, b / a=1$ and $\Omega=10$.

number of segments required to obtain an accurate value for the antenna current.

\section{B. Circular Loop-Comparison to Previous Work}

With the convergence properties of the model understood, we can now use it to investigate the correspondence between the results of this work and previously obtained results for circular loop antennas. 


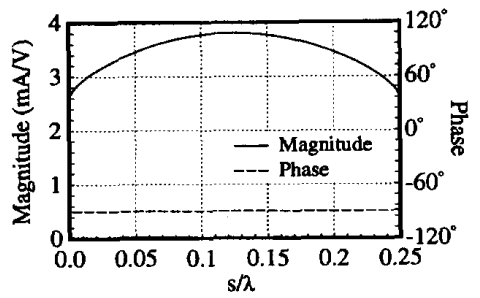

(a)
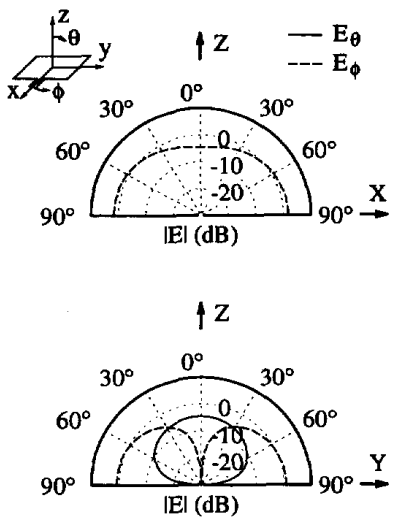

(b)

Fig. 6. (a) Current distribution (magnitude and phase) versus perimeter coordinate $s$ and (b) principal plane directivity patterns (in $\mathrm{dB}$ ) versus $\theta$ for a $0.25 \lambda$ loop with $\nu=10, b / a=1$ and $\Omega=10$.

Fig. 4 shows a typical plot of the antenna input impedance versus loop circumference $P$ in wavelengths for a circular loop antenna ( $\nu=2$ ) with $\Omega=10$ and $\Omega=12$. The magnetic frill generator was again applied here. Of interest in this plot is the antiresonant behavior which causes excessively large impedance values for circumferences near $\lambda / 2$. The dots in the figure correspond to values taken from computations of a previous work which uses the Fourier series approach with a magnetic frill generator for a loop with $\Omega=10$ [9]. Clearly, the results of the present study match very well with these established results. It should be noted that the computed values in [9] are shown in that work to compare very well with actual measured impedance data. Comparison of the plot of Fig. 4 with other similar plots appearing in past literature [3], [4] further shows that the model faithfully reproduces the results of previous studies.

\section{Current Distribution and Directivity Patterns}

Fig. 5(a) shows the current distribution (magnitude and phase) versus arc length $s$ from the feed point for the case of a superquadric loop with perimeter $P=0.75 \lambda, \nu=10, a=b$ and $\Omega=$ $2 \ln \left(P / r_{o}\right)=10$. The magnetic frill source model is used. As has been established in past studies, the current is nearly sinusoidal in nature, therefore dismissing the use of constant current models for predicting the loop radiation and impedance behavior. This variation in the current leads to the interesting far-field directivity patterns for the principal planes shown in Fig. 5(b). This figure plots the directivity in $\mathrm{dB}$ as a function of $\theta$ for a loop in the $x y$ plane as shown in the inset. The patterns for the principal planes are provided in the upper hemisphere $(z>0)$ only as the pattern is obviously

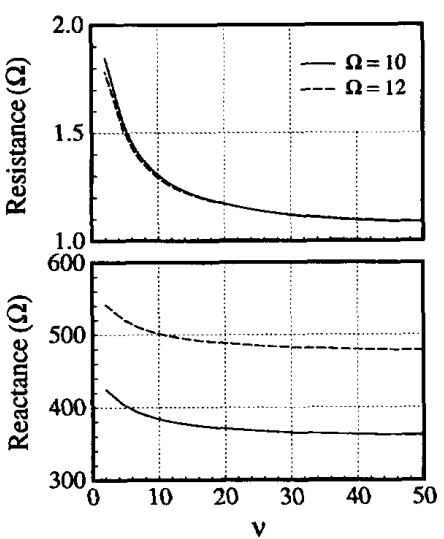

(a)

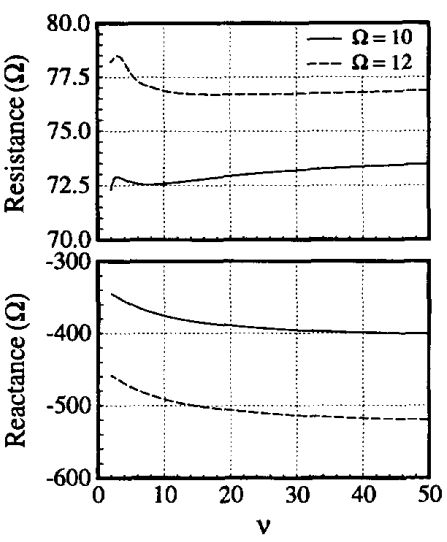

(b)

Fig. 7. Input impedance versus $\nu$ for a superquadric loop with $b / a=1$ for $\Omega=10$ and 12: (a) $0.25 \lambda$ loop, (b) $0.75 \lambda$ loop.

symmetric about the $x y$ plane. Figs. 6(a) and (b) show a similar result for a small $0.25 \lambda$ antenna also with $\nu=10, a=b$, and $\Omega=10$. Here, the current variation is much less pronounced, as has been demonstrated in previous work [6].

\section{Parametric Studies}

The flexibility of the superquadric representation allows investigation of the effect of geometrical parameters on the antenna characteristics. Figs. 7(a) and (b) provide one such study by presenting the input impedance versus squareness parameter $\nu$ for loops with total perimeters of $P=0.25 \lambda$ and $P=0.75 \lambda$ and with $a=b$. Results for $\Omega=10$ and $\Omega=12$ are provided for the $50 \Omega$ magnetic frill source. These plots show a noticeable variation in the input impedance, as the squareness of the loop increases. Fig. 8 illustrates the variation in input impedance versus the aspect ratio $b / a$ as the total perimeter is kept fixed at $0.25 \lambda$. In this case, the delta gap source model is used with a gap width of $0.01 \lambda$ for $\Omega=10$ and for two values of $\nu$. Once again, we see the effect of the shape of the loop on the antenna input impedance. Plots such as these can help in determining the most appropriate shape and size of the loop for matching purposes in antenna system design. 


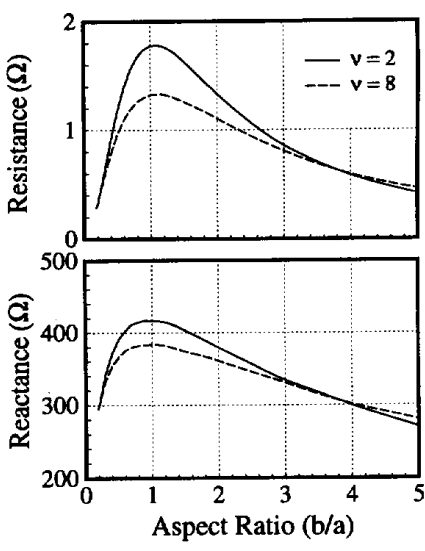

Fig. 8. Input impedance versus aspect ratio $b / a$ for a $0.25 \lambda$ loop with $\Omega=10$ for $\nu=2$ and 8 .

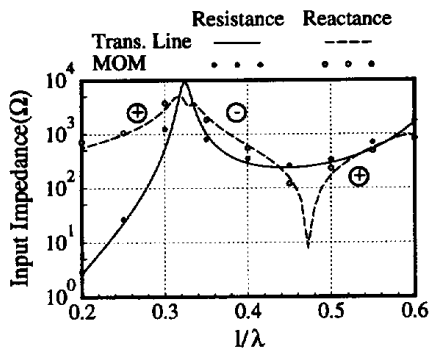

Fig. 9. Input impedance versus dipole length $l=2 b$ for a folded dipole with $r_{o}=0.0005 \lambda, \nu=20$ and wire spacing $2 a=0.00613 \lambda$ compared with the results obtained from a transmission line model.

\section{E. Folded Dipole}

As mentioned previously, the versatility of the superquadric representation for loop antennas allows modeling of folded dipoles through proper selection of the geometrical parameters. An example of such a computation is depicted in Fig. 9, which provides the input impedance versus dipole length $l=2 b$ for a folded dipole with wire radius $r_{o}=0.0005 \lambda$ and wire spacing of $2 a=0.00613 \lambda$, corresponding to a transmission line with characteristic impedance $Z_{o}=300 \Omega$. The squareness parameter is chosen to be $\nu=20$. The lines in this plot correspond to computations performed using the transmission line model for the folded dipole as described in [19] and [20]. As can be seen, our moment method results agree well with the analytical solution. It should be mentioned that very fine subsectioning is required in the moment method solution to accurately represent the small wire spacing (2a) and provide the accuracy depicted in the figure.

\section{CONCLUSION}

In this work we have presented the development of a superquadric model of loop radiators which allows for investigation of a large range of practical antennas, including circular, elliptical and rectangular geometries, using one unified analysis. We have presented a useful parametric representation for the superquadric geometry and have applied this transformation to enable solution of the integral equation formulation via a subsectional domain moment method.
Application of this construction allows the superquadric curve to be represented with curved rather than piecewise linear subsectional segments, resulting in a computationally efficient algorithm. The current distribution in the loop computed from the integral equation is subsequently used to obtain the antenna input impedance and directivity pattern. Several representative results have been given which not only provide insight into the effects of such factors as the loop squareness and perimeter on the antenna behavior but also show the model's versatility in analyzing many different types of structures. In many applications where the system configuration places constraints on the antenna shape and size, incorporation of this formulation in the design process can help in determining the most suitable radiator to meet the needs of the system. In a future correspondence, the analysis of two coupled superquadric loops with arbitrary relative positions and orientations will be presented [15].

\section{REFERENCES}

[1] H. C. Pocklington, "Electrical oscillations in wires," Proc. Cambridge Phil. Soc., vol. 9, pp. 324-332, 1897.

[2] E. Hallén, "Theoretical investigations into transmitting and receiving qualities of antennae," Nova Acta Regiae Soc. Sci. Ups., ser. 4, vol. 11, pp. 1-44, 1938.

[3] J. E. Storer, "Impedance of thin-wire loop antennas," Trans. Am. Inst. Electr. Eng., vol. 75, Pt. 1, (Communication and Electronics), pp. 606-619, Nov. 1956.

[4] C. W. Harrison and R. W. P. King, "Folded dipoles and loops," IRE Trans. Antennas Propag., vol. AP-9, pp. 171-187, Mar. 1961.

[5] T. T. Wu, "Theory of the thin circular loop antenna," J. Mathematical Phys., vol. 3, pp. 1301-1304, Nov.-Dec. 1962.

[6] R. W. P. King and G. S. Smith, Antennas in Matter: Fundamentals, Theory and Applications, Cambridge, MA: MIT Press, 1981, ch. 9.

[7] R. F. Harrington and J. Mautz, "Electromagnetic behaviour of circular wire loops with arbitrary excitation and loading," Proc. IEE, vol. 115, pp. 68-77, Jan. 1968.

[8] N. Inagaki, T. Sekiguchi and S. Ito, "A theory of a loop antenna," Electron. Commun. Japan, vol. 53-B, pp. 62-70, Mar. 1970.

[9] G. Zhou and G. S. Smith, "An accurate theoretical model for the thinwire circular half-loop antenna," IEEE Trans. Antennas Propag., vol. 39, pp. 1167-1177, Aug. 1991.

[10] S. Adachi and Y. Mushiake, "Theoretical formulation for circular loop antennas by integral equation method," Sci. Rep. Res. Inst. Tohoku Univ. ser. B, vol. 9, pp. 9-18, 1957.

[11] S. Adachi and Y. Mushiake, "Studies of large circular loop antennas," Sci. Rep. Res. Inst. Tohoku Univ. ser. B, vol. 9, pp. 79-103, 1957.

[12] A. Baghdasarian and D. J. Angelakos, "Scattering from conducting loops and solution of circular loop antennas by numerical methods," Proc. IEEE, vol. 53, pp. 818-822, Jan. 1965.

[13] R. F. Harrington, Field Computation by Moment Methods, New York: Macmillan Co., 1968.

[14] A. H. Barr, "Superquadrics and angle-preserving transformations," IEEE Comput. Graphics and Appl., vol. 1, pp. 11-23, Jan. 1981.

[15] M. A. Jensen and Y. Rahmat-Samii, "Characterization of electromagnetically coupled superquadric loop antennas for mobile communications applications," To appear in IEE Proc. $H$.

[16] N. J. Champagne II, J. T. Williams and D. R. Wilton, "The use of curved segments for numerically modeling thin wire antennas and scatterers," IEEE Trans. Antennas Propag., vol. 40, pp. 682-689, June 1992.

[17] A. J. Poggio and E. K. Miller, "Techniques for low-frequency problems," ch. 3 in Antenna Handbook: Theory, Applications and Design, Y. T. Lo and S. W. Lee, Eds., New York: Van Nostrand Reinhold Co. Inc., 1988.

[18] L. L. Tsai, "A numerical solution for the near and far fields of an annular ring of magnetic current," IEEE Trans. Antennas Propag,, vol. AP-20, pp. 569-576, Sept. 1972.

[19] G. A. Thiele, E. P. Ekelman, Jr. and L. W. Henderson, "On the accuracy of the transmission line model of the folded dipole," IEEE Trans. Antennas Propag., vol. AP-28, pp. 700-703, Sept. 1980.

[20] C. A. Balanis, Antenna Theory: Analysis and Design, New York: Harper \& Row, Publishers, 1982, pp. 340-346. 